\title{
Traditional remedies used in the Western Pacific for the treatment of ciguatera poisoning
}

\author{
G. Bourdy ${ }^{\mathrm{a}}$, P. Cabalion ${ }^{\mathrm{b}}$, P. Amade ${ }^{\mathrm{a}}$ and D. Laurent ${ }^{\mathrm{a}}$ \\ ${ }^{a}$ Laboratoire de Pharmacologie, Centre ORSTOM, B.P. A5, Nouméa (New Caledonia) and ${ }^{b} 9$, rue Suètone, 67200 Strasbourg \\ (France)
}

(Received June 23, 1991; accepted January 27, 1992)

\begin{abstract}
Ciguatera is a specific type of food poisoning associated with the ingestion of tropical fish, which, although normally safe for consumption, may at times contain high amounts of ciguatoxin, as well as other chemically related toxins. Widespread in tropical regions where coral reefs are present, ciguatera fish poisoning constitutes a major hindrance for local fishing industries, local economies and foreign trade. Because no symptomatic treatment has been totally satisfactory, folk remedies remain of great interest. In this paper, a list of plants used in treating ciguatera poisoning in the Western Pacific region is presented; with emphasis on species whose exact utilization (part of plant used, preparation, dosage) has been determined.
\end{abstract}

Key words: traditional remedies; ciguatera poisoning; Western Pacific

\section{Introduction}

The ciguatera toxins are produced by the benthic dinoflagellate Gambierdiscus toxicus (Bagnis et al., 1980). This single-celled microalgae is ingested by herbivorous fish along with the macrophytic algae on which it grows. The toxins are then passed up the food chain until they reach sufficient concentration to provoke a type of human poisoning known as ichthyosarcotoxism. This condition is difficult to prevent, since tainted fish cannot be identified by appearance, odor or taste. All large species of fish associated with coral reefs can be toxic, particularly those at the upper end of the food chain (barracudas, groupers, sea perch, moray eels, sharks, etc).

Several 'ciguateric' toxins, some not yet identified, are involved in the etiology of ciguatera fish poisoning. The two major toxins are ciguatoxin (CTX) and maitotoxin (MTX) (Ohizumi, 1987). The chemical structure of ciguatoxin has recently been determined (Murata et al., 1989). It is one of the most potent marine toxins with a $50 \%$ lethal dose $\left(\mathrm{LD}_{50}\right)$ in mice of $0.45 \mu \mathrm{g} / \mathrm{kg}$ when injected intraperitoneally (i.p.) and cannot be destroyed by cooking or freezing. Maitotoxin, which can be biosynthesized in cultures of Gambierdiscus toxicus (Yasumoto et al., 1987), is particularly toxic $\left(\mathrm{LD}_{50}=0.13 \mu \mathrm{g} / \mathrm{kg}\right.$, i.p.) and is found only in the

Correspondence to: G. Bourdy, Laboratoire de Pharmacologie, Centre ORSTOM, B.P. A5, Nouméa, New Caledonia. viscera of herbivorous and bottom-feeding fish (Yokoyama et al., 1988).

Symptoms of poisoning appear $2-30 \mathrm{~h}$ after ingestion of toxic fish. Early signs include numbness, a prickling sensation in the lips, tongue and throat, general weakness and nausea. These symptoms soon give way to digestive (cramps, abdominal pains, vomiting and profuse diarrhea) and neurological disorders: itching (pruritus), articular pains and asthenia. In severe cases, cardiovascular and respiratory symptoms may also occur.

The severity of the symptoms varies according to the amount of toxin ingested (based on the fish size and the part of the fish consumed) and individual susceptibility.

In most cases of ciguatera fish poisoning, complete recovery may be expected, but exceptionally severe poisoning can lead to paralysis, coma and death.

\section{Mode of action of ciguatoxin}

CTX modifies cell membrane permeability to sodium ions: it opens $\mathrm{Na}^{+}$channels in the nerves of intact mammals, leading to an influx of intracellular sodium and depolarization of the nerve fibre (Capra and Cameron, 1985).

Studies by Bidard et al. (1984) and Lombet et al. (1987) have further clarified CTXs mode of action: it acts at protein sites of tetrodotoxin-sensitive sodium channels. The activation of these sites leads to the opening of fast sodium channels. 
Ciguatoxin and brevetoxin (toxin implicated in neurotoxic shellfish poisoning) share the same site of action, which has been shown to be distinct from that used by other toxins acting on fast sodium channels (tetrodotoxin, saxitoxin, veratridine, batrachotoxin, aconitine, grayanotoxin, scorpion and sea anemone toxins, pyrethroids and DDT).

Membrane depolarization resulting from the influx of sodium brings about an opening of calcium channels and therefore an influx of $\mathrm{Ca}^{2+}$, which in turn activates the release of sympathetic noradrenaline and parasympathetic (acetylcholine) transmitters.

Disorders observed after ingestion of CTX, therefore, result from an intermingling of adrenergic and cholinergic effects of variable intensity.

\section{Treatment}

In most cases, ciguatera fish poisoning has no lasting effect. When the condition is identified, however, patients are advised to avoid ingestion of fish or other seafood as well as alcoholic beverages, nuts and grains, since these can aggravate symptoms or slow down recovery.

Up to now, treatment for ciguatera poisoning has consisted of methods aimed at reducing symptoms: stomach pumping and early administration of apomorphine. Subsequent treatment depends on the severity of each case and may include antispasmodics and antiemetics, for digestive disorders and vitamins $\mathbf{B}_{1}, \mathbf{B}_{6}, \mathbf{B}_{12}$ complex, colchicine, acetylsalicylic acid and calcium gluconate injections, for neurological symptoms.

Pruritus is treated with synthetic antihistamines. In severe cases, cardiovascular stimulants are administered to counter shock or collapse.

Alongside this approach of treatment, recent advances in knowledge of CTXs mode of action have led to treatments using various antagonists. Among the most promising are atropine-type substances, which counteract the effects associated with acetylcholine secretion. Such substances include lidocaine and tocainide (contact anesthetics with membrane stabilizing action which act as competitive antagonists with the sodium ion), phentolamine (an alpha-blocking substance) and calcium gluconate (Legrand et al., 1985; Lange et al., 1988). Clinical tests using intravenous mannitol have also been conducted with promising results (Palafox et al., 1988).

No symptomatic treatment has been totally satisfactory and many people continue to resort to healers and traditional medicines. Some of these preparations appear to have effective therapeutic properties.

Folk remedies, therefore, remain of great interest. In many places, there are no clinics close to villages and people must treat themselves with their own time-honoured recipes. It is these type of treatments which we have attempted to document.

\section{Methodology}

The ethnobotanical data was gathered over a period of 10 years $(1980-1990)$ by way of general ethnopharmacological surveys conducted on the various islands in the Vanuatu archipelago (G. Bourdy, A. Walter and P. Cabalion) and in New Caledonia (D. Bourret, P. Amade and D. Laurent). People from all walks of life were invited to contribute their recipes for remedies used to prevent or treat ciguatera fish poisoning. In this way, some recipes came from ordinary men and women, whereas others were volunteered by traditional doctors or by specialists. Specimens of the plant species listed below were collected with the help of these informants. Wherever possible, four specimens of each species were prepared and sent to each of the following institutions: National Museum of Natural History (Paris, France), the ORSTOM Herbarium (Nouméa, New Caledonia), the Kew Gardens Herbarium (Surrey, England) and the National Collection (Port Vila, Vanuatu). All identifications were confirmed by specialists.

\section{Results}

The results of the 10-year survey are summarized in Table 1. In this table, we have indicated the plants' species and family names and their common French names, as well as their Herbarium reference numbers, whenever these could be obtained. Plants used to treat ciguatera fish poisoning are clearly indicated and distinguished from those used to treat other types of fish poisoning. The mode of preparation and the dosage, if known, are also indicated.

Some of the information gathered here has already been published; when this is the case, the source reference is given in parentheses. If the source of data is our own observations, this is indicated by an asterisk.

In addition to methods used to treat ciguatera fish poisoning in Vanuatu and New Caledonia, those used in other Pacific countries (French Polynesia, Guam, Samoa, Tonga, Fiji) were also considered. 
TABLE 1

PLANTS USED FOR THE TREATMENT OF CIGUATERA FISH POISONING

\begin{tabular}{|c|c|c|c|c|c|}
\hline $\begin{array}{l}\text { Species (Family) } \\
\text { French name used in New } \\
\text { Caledonia } \\
\text { English common name } \\
\text { Herbarium number }\end{array}$ & $\mathrm{Use}^{\mathrm{a}}$ & $\begin{array}{l}\text { Plant part } \\
\text { used }\end{array}$ & $\begin{array}{l}\text { Preparation, dosage (References) } \\
(*)=\text { Data derived from field observations and } \\
\text { interviews }\end{array}$ & $\begin{array}{l}\text { Frequency } \\
\text { and } \\
\text { Origin } b\end{array}$ & $\begin{array}{l}\text { Other uses }{ }^{\mathrm{C}} \text { (References) } \\
(*)=\text { Data derived from field } \\
\text { observations and interviews }\end{array}$ \\
\hline $\begin{array}{l}\text { Achyranthes aspera L. } \\
\text { (AMARANTHACEAE) }\end{array}$ & Ciguatera & Whole plant & Decoction (Rageau, 1973) & N.C. & $\begin{array}{l}\text { Relief of dysenteric symptoms; diuretic } \\
\text { (Rageau, 1973) }\end{array}$ \\
\hline GB507 & Ciguatera & Root & $\begin{array}{l}\text { Macerate in a bottle of water } \\
\text { Drink one bottle a day until recovery is complete }(*)\end{array}$ & $\begin{array}{l}\text { (3) } \\
\text { N.C. } \\
\text { Van. }\end{array}$ & $\begin{array}{l}\text { Antispasmodic (Rageau, 1973); relief } \\
\text { of dysenteric symptoms }\left(^{*}\right)\end{array}$ \\
\hline $\begin{array}{l}\text { Aglaia elaeagnoidea } \\
\text { (Jussieu) Bentham } \\
\text { (MELIACEAE) } \\
\text { "Bois rose" } \\
\text { PC1462 }\end{array}$ & Ciguatera & - & $\begin{array}{l}\text { Used in mixture with the following : } \\
\text { Maesa ambrymensis, Erythrina variegata, Metrosideros collina } \\
\text { var. villosa, Pandanus sp., Terminalia catappa,Dysoxylum } \\
\text { bijugum, Dysoxylum gaudichaudianum (Cabalion 1984e) }\end{array}$ & (1) & - \\
\hline $\begin{array}{l}\text { Aglaia saltatorum } \\
\text { A.C. Smith } \\
\text { (MELIACEAE) }\end{array}$ & Ciguatera & Stem & $\begin{array}{l}\text { The stem is scraped and squeezed into water in the preparation } \\
\text { of a remedy for fish poisoning (Weiner, 1985) }\end{array}$ & $\begin{array}{l}(1) \\
\text { Fiji }\end{array}$ & $\begin{array}{l}\text { Bark infusion is taken as an emetic } \\
\text { (Whistler, 1991) }\end{array}$ \\
\hline \multirow[t]{4}{*}{$\begin{array}{l}\text { Argusia argentea } \\
\text { (L.f.) Heine } \\
\text { (BORAGINACEAE) } \\
\text { "Faux tabac" } \\
\text { PC2282 }\end{array}$} & Ciguatera & Leaf & $\begin{array}{l}\text { One large leaf in an infusion or decoction. Use internally and } \\
\text { externally (baths) (Bourret, 1981)(*) }\end{array}$ & $\begin{array}{l}\text { (3) } \\
\text { N.C. } \\
\text { Van. } \\
\text { Pol. }\end{array}$ & $\begin{array}{l}\text { Muscle pain relief; antirheumatic } \\
\text { (Rageau, 1983); antipruritic (Geremie, } \\
\text { 1988; Rageau, 1973); depurative } \\
\text { (Pétard, 1986) }\end{array}$ \\
\hline & Fish poisoning & Leaf & $\begin{array}{l}\text { Leaf infusion is taken as an antidote for poisoning caused by } \\
\text { eating tainted fish (Whistler, 1991) }\end{array}$ & Tonga & Antipruritic (Bourret, 1981) \\
\hline & Ciguatera & Stembark & $\begin{array}{l}\text { Boil a piece of the stembark the size of the palm of the hand } \\
\text { in a litre of water for } 10 \text { minutes (Bourret, 1981; Cabalion, } \\
\text { 1984b; Pétard, 1986)(*) }\end{array}$ & & - \\
\hline & Ciguatera & Root & (Rageau, 1983) & (1) & - \\
\hline
\end{tabular}


TABLE 1 (continued)

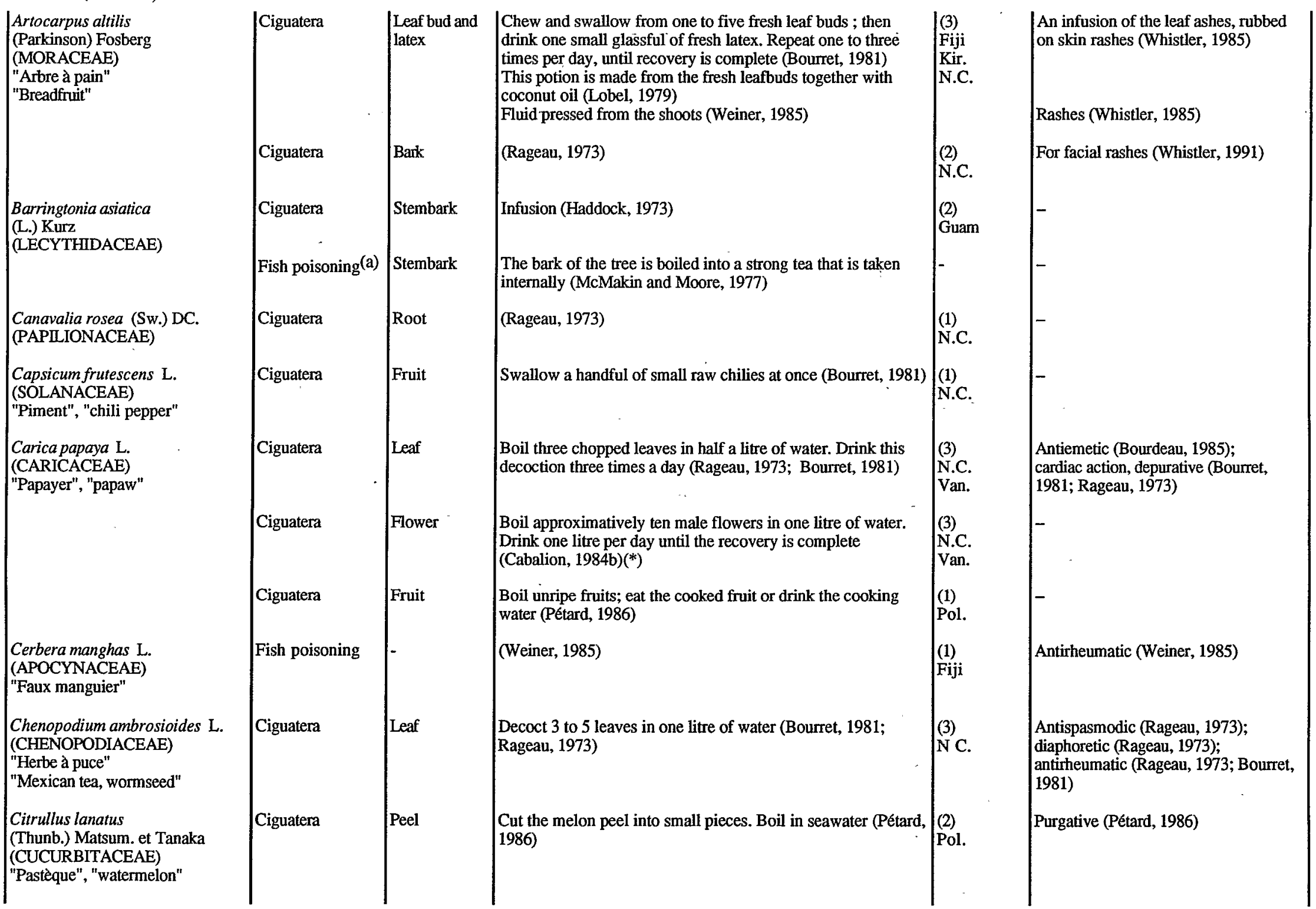




\begin{tabular}{|c|c|c|c|c|c|}
\hline $\begin{array}{l}\text { Clerodendrum inerme } \\
\text { (L.) Gaertner } \\
\text { (VERBENACEAE }\end{array}$ & Fish poisoning & Seed & Decoct freshly pounded seeds (Weiner, 1971) & (1) & - \\
\hline $\begin{array}{l}\text { Cocos nucifera L. } \\
\text { (ARECACEAE) } \\
\text { "Cocotier", "coconut tree" }\end{array}$ & Fish poisoning & Fruit & $\begin{array}{l}\text { Coconut milk, produced by grating and squeezing the flesh, is } \\
\text { used to treat fish poisoning (Weiner, 1985) } \\
\text { Mix coconut cream (obtained by scraping two coconuts) with } \\
\text { cocoa and sugar to a consistency of molasses. Drink one cup } \\
\text { (McCuddin, 1974) }\end{array}$ & $\begin{array}{l}(3) \\
\text { Fiji } \\
\text { Samoa }\end{array}$ & $\begin{array}{l}\text { Emetic (McCuddin, 1974), } \\
\text { Coconut oil is a purgative (Pétard, } \\
\text { 1986) }\end{array}$ \\
\hline & Ciguatera & $\begin{array}{l}\text { Fluff } \\
\text { (mesocarp) }\end{array}$ & $\begin{array}{l}\text { Drink during three days the juice from the fluff (mesocarp) of } \\
\text { the coconut ("ovisi" or "nia" stage). This drink is claimed to } \\
\text { induce vomiting, which increase the elimination of the poison } \\
\text { (Pétard, 1986) }\end{array}$ & $\begin{array}{l}(1) \\
\text { Pol. }\end{array}$ & - \\
\hline $\begin{array}{l}\text { Coix lacryma-jobi L. } \\
\text { (POACEAE) } \\
\text { "Larmes de Job" }\end{array}$ & Ciguatera & Root & Infusion (Rageau 1973) & (1) & - \\
\hline $\begin{array}{l}\text { Crinum spp. } \\
\text { (AMARYLLIDACEAE) } \\
\text { "Lys sauvage" }\end{array}$ & Ciguatera & Bulb & (Rageau, 1973) & (2) & $\begin{array}{l}\text { Purgative, diuretic, emetic, diaphoretic } \\
\text { (Rageau, 1973) }\end{array}$ \\
\hline $\begin{array}{l}\text { Davallia solida } \\
\text { (J.R. et G. Forster) Swartz } \\
\text { (DAVALLIACEAE) } \\
\text { PC1012 }\end{array}$ & Ciguatera & Rhizome & Same recipes as for D. epiphylla (same references) & $\begin{array}{l}\text { (3) } \\
\text { N.C. }\end{array}$ & $\begin{array}{l}\text { Analgesic (Vienne, 1981); purgative } \\
\text { (Whistler, 1985) }\end{array}$ \\
\hline $\begin{array}{l}\text { Duboisia myoporoides } \mathrm{R} . \mathrm{Br} \text {. } \\
\text { (APOCYNACEAE) } \\
\text { "Bois bouchon" }\end{array}$ & Ciguatera & Leaf & Infuse young leaves (Barrau, 1950 ; Dufva et al., 1973) & $\begin{array}{l}(2) \\
\text { N.C. }\end{array}$ & - \\
\hline $\begin{array}{l}\text { Dysoxylum bijugum } \\
\text { (Labill.) Seemann } \\
\text { PC1963b and } \\
\text { Dysaxylum gaudichaudianum } \\
\text { (Juss.) Miq. } \\
\text { PC2268 } \\
\text { (MELIACEAE) }\end{array}$ & Ciguatera & Stembark & $\begin{array}{l}\text { See Aglaia elaeagnoidea } \\
\text { Use the stembark of these two Dysoxylum spp. together with } \\
\text { the stembark of Pandanus sp. and the twigs of Terminalia } \\
\text { catappa }\left(^{*}\right)\end{array}$ & $\begin{array}{l}(1) \\
\text { Van. } \\
(1) \\
\text { Van. }\end{array}$ & - \\
\hline
\end{tabular}


TABLE 1 (continued)

\begin{tabular}{|l} 
Elattostachys falcata (A. Gray) \\
Radlk. \\
(SAPINDACEAE) \\
Elephantopus mollis H.B. K. \\
(ASTERACEAE) \\
Entada phaseoloides (L.) Merrill \\
(MIMOSACEAE) \\
"Water vine" \\
Erythrina fusca Lour. \\
(PAPILIONACEAE) \\
Erythrina variegata L. \\
(PAPILIONACEAE) \\
"Peuplier canaque", "coral tree" \\
\\
Euphorbia hirta L. \\
(EUPHORBIACEAE) \\
"Herbe à dysenterie" \\
"Hairy spurge" \\
Ficus habrophylla \\
G. Bennett ex Seemann and \\
Ficus aspera Forster f. \\
Ficus sp. \\
(MORACEAE) \\
Hernandia nymphaeifolia \\
(Presl) Kubitzki \\
(HERNANDIACEAE) \\
Inocarpus fagifer (Parkinson) \\
Fosberg \\
(PAPIIONACEAE) \\
"Chataîgnier de Tahiti", \\
"Tahitian chestnut tree" \\
Ipomoea pes-caprae \\
(L.) R. Brown \\
(CONVOLVULACEAE) \\
"Liseron de mer" \\
"Beach morning glory vine" \\
\end{tabular}

\begin{tabular}{|l|l} 
Fish poisoning & Leaf \\
Ciguatera & - \\
Cish poisoning & Stem \\
Ciguatera & Stembark \\
Ciguatera & Sish poisoning \\
Ciguatera & Leaf \\
Cish poisoning & Leat \\
Reat
\end{tabular}

Juice pressed from the leaves is used as an antidote to
ingestion of poisonous fish (Weiner, 1985)
Decoction (Vienne, 1981)
Crush the stem with a small amount of water (Weiner, 1985)
Macerate (Rageau, 1973)
Macerate (Rageau, 1973)
Use with the bark of Pterocarpus indicus. Grind the barks, add
water and drink the juice (Cabalion, 1984e)
See Aglaia elaeagnoidea
Decoction. Do not exceed $1 \mathrm{~g}$ of dried plant per day (Rageau,
1973; Rageau, 1983)
Press the juice of the leaves (Weiner, 1985)
Cook the fish with the leaves; their sap is said to detoxify the
fish poison (Bourret, 1981)
Warm the leaves over a fire then press to extract the juice;
drink this juice (Cabalion, 1984e)
Chew the roots (Petard, 1986)
(Cabalion, 1984e)

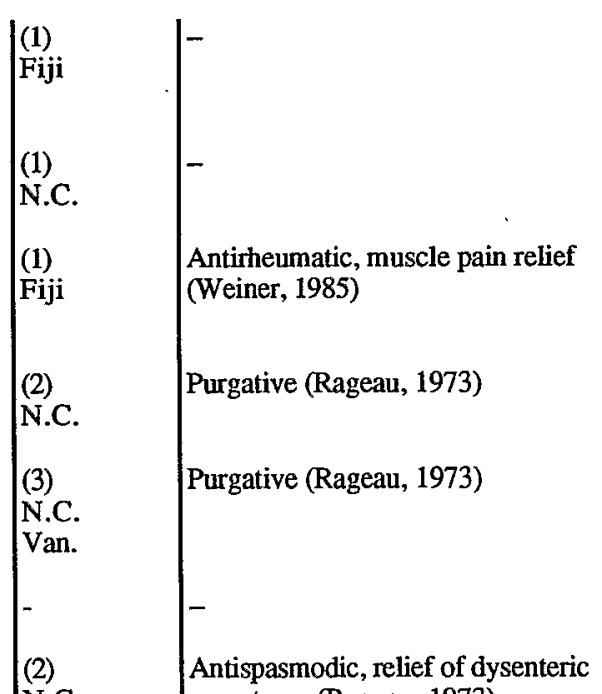

symptoms (Rageau, 1973) 


\begin{tabular}{|l} 
Maba buxifolia \\
Pers. (Doubtfull name) \\
(EBENACEAE) \\
Macropiper latifolium \\
(L.f.) Miquel \\
(PIPERACEAE) \\
"Faux kava" \\
AW126 \\
Maesa ambrymensis Guillaumin \\
(MYRSINACEAE) \\
Maesa insularis Gillespie \\
(MYRSINACEAE) \\
Metrosideros collina \\
(J.R. et G. Forster) A. Gray \\
var. villosa (L.f.) A. Gray \\
(MYRTACEAE) \\
Microsorium punctatum' \\
(L.) Copeland \\
(POLYPODIACEAE) \\
Morinda citrifolia L. \\
(RUBIACEAE) \\
"Fromager" \\
"Indian mulberry" \\
Pandanus tectorius Parkinson \\
(PANDANACEAE) \\
"Pandanus" \\
"Screw pine" \\
AW104 \\
Phaleria glabra \\
(Turrill) Domke \\
(THYMELAEACEAE) \\
Pandanus sp. \\
(PANDANACEAE) \\
PC2262 \\
\end{tabular}

\begin{tabular}{|c|c|c|}
\hline Ciguatera & $\begin{array}{l}\text { Leaf, } \\
\text { Stembark }\end{array}$ & Prepare a decoction from the leaves and bark (Rageau, 1973) \\
\hline Ciguatera & Fruit & Eat 4 to 5 uncooked fruits. Repeat if symptoms persist $\left(^{*}\right)$ \\
\hline Ciguatera & - & See Aglaia elaeagnoidea \\
\hline Fish poisoning & Stembark & $\begin{array}{l}\text { The bark is scraped, mixed with milk from the red-skinned } \\
\text { coconut and drunk (Weiner, 1985) }\end{array}$ \\
\hline Ciguatera & - & See Aglaia elaeagnoidea \\
\hline Ciguatera & - & (Rageau, 1973) \\
\hline Ciguatera & Fruit & $\begin{array}{l}\text { Crush together } 3 \text { unripe and } 3 \text { ripe fruits, then extract the } \\
\text { juice. Mix with coconut milk and drink (Petard, 1986) }\end{array}$ \\
\hline Ciguatera & Aerial root & $\begin{array}{l}\text { Scrape the inside of an aerial root and press out a teaspoonfull } \\
\text { of juice. Drink a teaspoonfull of juice three times a day, until } \\
\text { recovery is complete }\left({ }^{*}\right)\end{array}$ \\
\hline Ciguatera & Leaf & Drink the juice pressed from young leaves $\left(^{*}\right)$ \\
\hline Ciguatera & - & See Aglaia elaeagnoidea \\
\hline Ciguatera & Bark & $\begin{array}{l}\text { See Dysoxylum bijugum and } \\
\text { D. gaudichaudianum }\end{array}$ \\
\hline Fish poisoning & Aerial root & $\begin{array}{l}\text { Fluid is pressed from the above ground prop root (Weiner, } \\
1985 \text { ) }\end{array}$ \\
\hline Fish poisoning & Leaf & Juice is pressed from the leaves (Weiner, 1985) \\
\hline
\end{tabular}

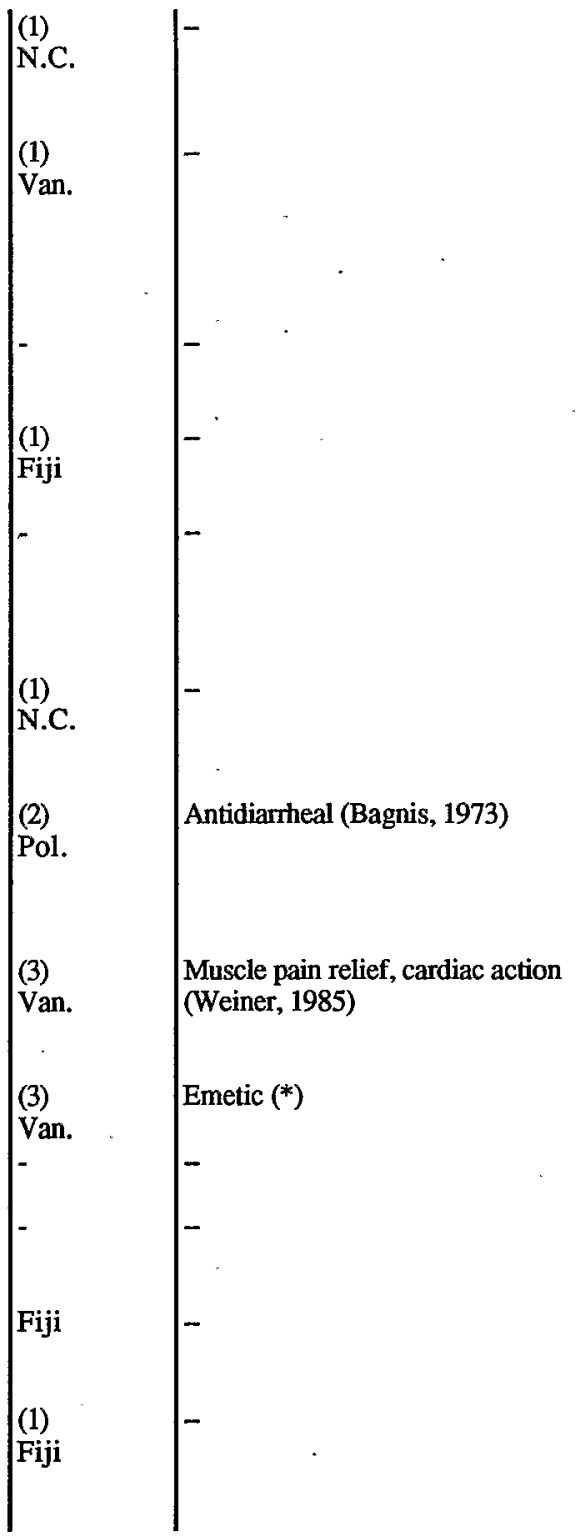


TABLE 1 (continued)

\begin{tabular}{|l} 
Phymatosorus nigrescens \\
(Blume) Copeland \\
(POLYPODIACEAE) \\
Phymatosorus scolopendria \\
(Burman f.) Pichi-Sermolli \\
(POLYPODIACEAE) \\
\\
Pithecellobium dulce \\
(Roxburgh) Bentham \\
(MIMOSACEAE) \\
"Pois doux", "Madras thom" \\
Plectranthus parviflorus Willd. \\
(LABLATAE) \\
AL1 \\
Polyscias guilfoylei \\
(Bull.) L.H. Bailey \\
(ARALIACEAE) \\
AL2 \\
Polyscias scutellaria \\
(Burman f.) Fosberg \\
(ARALIACEAE) \\
AL3 \\
Pterocarpus indicus Willdenow \\
(PAPILIONACEAE) \\
"Narra tree, New guinea \\
rosewod" \\
GB879, PC1308 \\
Santalum austro-caledonicum \\
Vieillard \\
(SANTALACEAE) \\
"Bois de santal", "sandalwood" \\
\end{tabular}

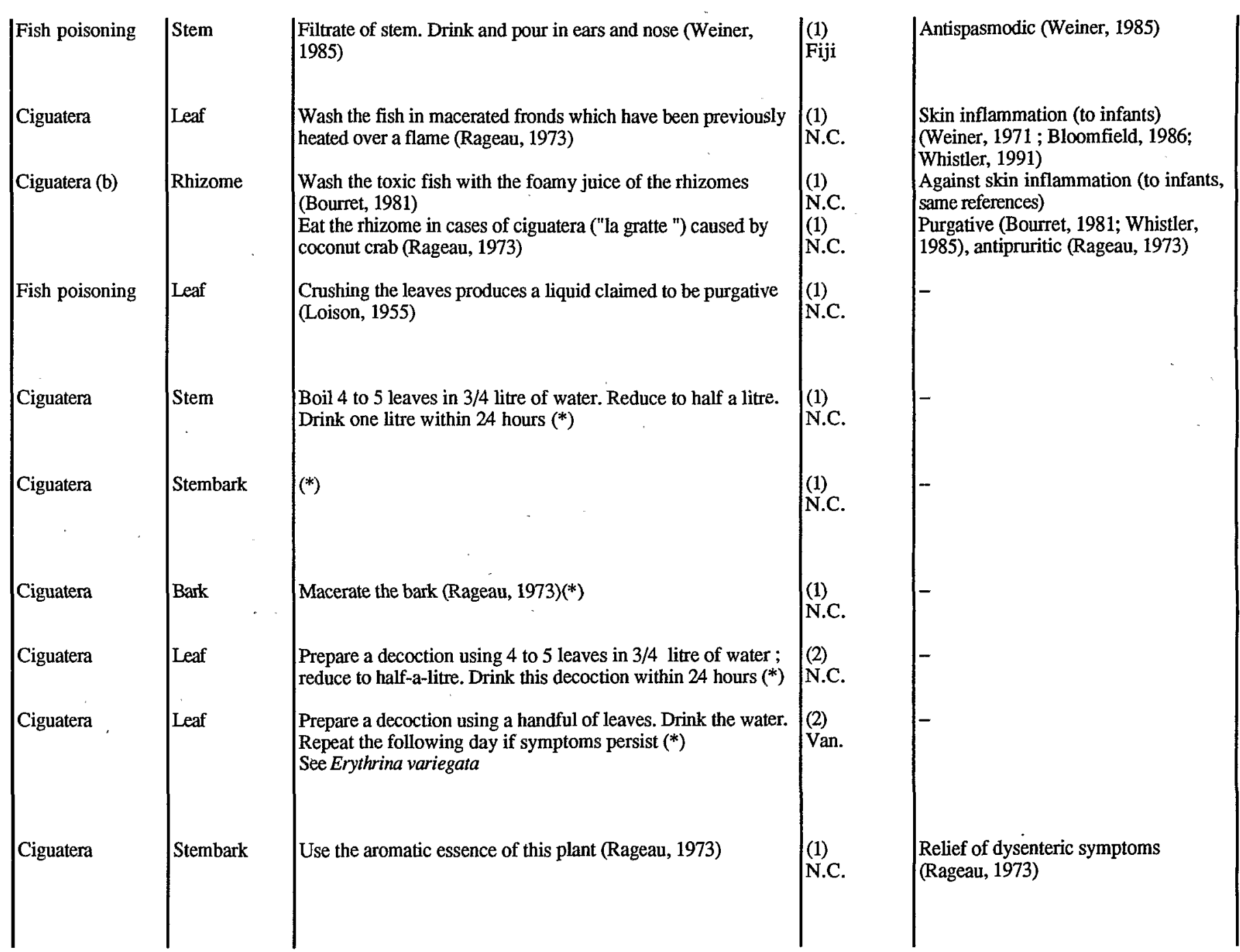




\begin{tabular}{|l} 
Scaevola montana Labillardière \\
(GOODENIACEAE) \\
Scaevola neoebudica Guillaumin \\
(GOODENIACEAE) \\
PC2594 \\
Scaevola sericea Vahl \\
(GOODENICEAE) \\
Schinus terebenthifolius Raddi \\
(ANACARDIACEAE) \\
"Faux poivrier","Christmas berry" \\
AL4 \\
Senna occidentalis (L.) Link \\
(CAESALPINIACEAE) \\
"Casse puante" \\
Sophora tomentosa L. \\
(PAPILIONACEAE) \\
Spondias cytherea \\
Sonnerat \\
(ANACARDIACEAE) \\
"Pomme cythère", \\
"Polynesian plum" \\
AW21, PC572, CS355 \\
\\
Stachytarpheta urticaefolia \\
(Salisb.) Sims \\
(VERBENACEAE) \\
"Herbe bleue" \\
AL9 \\
Syzygium malaccense \\
(L.) Merrill et Perry \\
(MYRTACEAE) \\
CS376 \\
"pomme canaque", "malay apple" \\
\end{tabular}

\begin{tabular}{|c|c|c|}
\hline |Ciguatera & Leaf & (Use the young leaves (Rageau, 1973) \\
\hline Ciguaterā & Leaf & $\begin{array}{l}\text { Crush a handful of leaves until you obtain a small glassful of } \\
\text { juice. Drink one glass per day ; repeat if necessary }\left({ }^{*}\right)\end{array}$ \\
\hline Ciguatera & Fruit & $\begin{array}{l}\text { Crush the fruit ; mix with coconut milk. Drink this } \\
\text { preparation (Petard, 1986) }\end{array}$ \\
\hline Ciguatera & Leaf & $\begin{array}{l}\text { Prepare an infusion from a bunch of leaves in one litre of } \\
\text { water }\left(^{*}\right)\end{array}$ \\
\hline Ciguatera & Leaf & Eat the uncooked leaves (Bourret, 1981) \\
\hline Ciguatera & & Eat 3 to 7 seeds (Pétard, 1986) \\
\hline Ciguatera & Leaf & $\begin{array}{l}\text { Maceration (Vienne, 1981) } \\
\text { Express the juice from the leaves to obtain a small glassful o } \\
\text { juice. Drink one-half glass per day until recovery is complete } \\
\left(\left(^{*}\right)\right. \\
\text { Prepare a decoction from the leaves. Drink one-half glass per } \\
\text { day until recovery is complete }\left({ }^{*}\right)\end{array}$ \\
\hline Ciguatera & Stembark & $\begin{array}{l}\text { Grind the inside of the bark and express the juice from it. } \\
\text { Drink from } 3 \text { to } 4 \text { small glassfuls of juice per day until } \\
\text { recovery is complete (Weiner, 1985) }\end{array}$ \\
\hline Ciguatera & Root & Maceration (Bourret, 1981) \\
\hline Ciguatera & Stembark & $\begin{array}{l}\text { Decoction (Rageau, 1973) } \\
\text { Prepare a small glassful of the concentrated juice from the } \\
\text { inner bark; drink three small glassfuls per day ; repeat if } \\
\text { necessary }\left({ }^{*}\right)\end{array}$ \\
\hline
\end{tabular}

\begin{tabular}{|c|c|}
\hline $\begin{array}{l}(1) \\
\text { N.C. }\end{array}$ & - \\
\hline $\begin{array}{l}\text { (2) } \\
\text { Van. }\end{array}$ & - \\
\hline (1) & - \\
\hline $\begin{array}{l}\text { (1) } \\
\text { N.C. }\end{array}$ & Antirheumatic (Rageau, 1973) \\
\hline $\begin{array}{l}(1) \\
\text { N.C. }\end{array}$ & $\begin{array}{l}\text { Diaphoretic (Bourret, 1981) } \\
\text { Depurative }\left({ }^{*}\right)\end{array}$ \\
\hline $\begin{array}{l}\text { (1) } \\
\text { Pol. }\end{array}$ & $\begin{array}{l}\text { Antidiarrheal, relief of dysenteric } \\
\text { symptoms, antiemetic (Pétard, 1986) }\end{array}$ \\
\hline $\begin{array}{l}\text { (3) } \\
\text { Van. }\end{array}$ & Emetic (Vienne, 1981) \\
\hline $\begin{array}{l}\text { (3) } \\
\text { Fiji }\end{array}$ & - \\
\hline $\begin{array}{l}\text { (1) } \\
\text { N.C. }\end{array}$ & Diaphoretic (Bourret, 1981) \\
\hline $\begin{array}{l}\text { (3) } \\
\text { N.C. } \\
\text { Van. }\end{array}$ & $\begin{array}{l}\text { Depurative, laxative (Bourret, 1981), } \\
\text { antirheumatic }\left(^{*}\right)\end{array}$ \\
\hline
\end{tabular}


TABLE 1 (continued)

\begin{tabular}{|c|c|c|c|c|c|}
\hline $\begin{array}{l}\text { Terminalia catappa L. } \\
\text { (COMBRETACEAE) }\end{array}$ & Ciguatera & 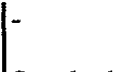 & See Aglaia elaeagnoidea & - & $\begin{array}{l}\text { Cardiac action (Bourret, 1981), emetic } \\
\text { in infants (Whistler,1991) }\end{array}$ \\
\hline "Badamier", & Ciguatera & Stembark & $\begin{array}{l}\text { See Dysoxylum bijugum and D. gaudichaudianum } \\
\text { Cut out a sheet of stembark } 5 \text { by } 10 \mathrm{~cm} \text { in size; boil in one } \\
\text { litre of water until a light red decoction is obtained; drink one } \\
\text { glassful } 2 \text { to } 3 \text { times a day (Bourret, 1981) }\end{array}$ & $\begin{array}{l}- \\
(2) \\
\text { N.C. } \\
\text { Van. }\end{array}$ & \\
\hline $\begin{array}{l}\text { Thespesia populnea (L.) } \\
\text { Solander ex Correa } \\
\text { (MALVACEAE) } \\
\text { "Bois de rose", "milo tree" }\end{array}$ & Ciguatera & $\begin{array}{l}\text { Fruit, } \\
\text { Bark }\end{array}$ & $\begin{array}{l}\text { Juice of the unripe fruit and decoction of the bark orally } \\
\text { (Rageau, 1973) } \\
\text { Decoction (Bourdeau 1985) }\end{array}$ & $\begin{array}{l}(2) \\
\text { N.C. }\end{array}$ & $\begin{array}{l}\text { Relief of dysenteric symptoms } \\
\text { (Rageau, 1973) } \\
-\end{array}$ \\
\hline $\begin{array}{l}\text { Vitex rotundifolia } \\
\text { L.f Moldenke } \\
\text { (VERBENACEAE) } \\
\text { PC } 3092 \\
\text { "Gattilier" }\end{array}$ & Ciguatera & Root & $\begin{array}{l}\text { Maceration and decoction; use internally and externally (baths) } \\
\text { Rageau, 1973; Bourret, 1981; Vienne, 1981; Cabalion, } \\
\text { 1984e)(*) } \\
\text { Decoction; use intemally (same references) }\end{array}$ & $\begin{array}{l}\text { (3) } \\
\text { N.C. } \\
\text { Van. } \\
\text { (1) } \\
\text { N.C. }\end{array}$ & $\begin{array}{l}\text { Antiallergic (Rageau, 1973); } \\
\text { diaphoretic (Burkill, 1935) }\end{array}$ \\
\hline $\begin{array}{l}\text { Wikstroemia indica } \\
\text { (L.) C.A. Meyer } \\
\text { (THYMELAEACEAE) } \\
\text { GB1218 }\end{array}$ & Ciguatera & $\begin{array}{l}\text { Stembark } \\
\text { Leaf, bark }\end{array}$ & $\begin{array}{l}\text { Rub the entire body with the inside part of the bark. Repeat } \\
\text { these body rubs several times a day }\left(^{*}\right) \\
\text { (Rageau, 1983; Pétard, 1986) }\end{array}$ & $\begin{array}{l}(1) \\
\text { Van. } \\
(3) \\
\text { Pol. }\end{array}$ & $\begin{array}{l}\text { Purgative, emetic; species reputed to } \\
\text { be highly toxic (Rageau, } 1983 \text {; } \\
\text { Petard, 1986) }\end{array}$ \\
\hline $\begin{array}{l}\text { Ximenia americana } \mathrm{L} . \\
\text { (OLACACEAE) } \\
\text { "prune de mer" } \\
\text { Al8 }\end{array}$ & Ciguatera & Bark & (Legrand et al., personal communication) & (1) & - \\
\hline $\begin{array}{l}\text { Zingiber zerumbet } \\
\text { (L.) Roscoe ex J. E. Smith } \\
\text { (ZINGIBERACEAE) } \\
\text { "Gingembre". "shampoo ginger" }\end{array}$ & Fish poisoning & Rhizome & Fluid pressed from the rhizome (Weiner, 1985) & Fiji & - \\
\hline
\end{tabular}

'Use column: We made a distinction between ciguatera and other fish poisoning. (a) In some references, the term 'fish poisoning' appears; in such a'case, it is not possible to state whether or not the poisoning is due to the ciguateric toxin; for this reason, we have retained the term 'fish poisoning'. (b) In other cases, ciguatera poisoning may be caused by non ciguateric toxins, despite the author's use of the term 'la gratte', or ciguatera.

brequency and Origin column: We noted in this column a rough estimation of the frequency of use of the remedies listed and their geographical origin. (1) Recipe mentioned only once during the interviews or in the literature; (2) recipe mentioned twice or more; (3) recipe in general use. Kir., Kiribati; N.C., New Caledonia; Pol., Polynesia; Van., Vanuatu.

'Other Uses column: We noted in this column other properties of the plants when they were also used to treat some symptomatic effects of ciguatera (antiemetic, antispasmodic, relief of dysenteric symptoms, antidiarrheal, against skin inflammation, antiallergic, analgesic, muscle pain relief, antirheumatic, against rashes/antipruritic, cardiac action) or were likely to increase the elimination of the toxin (depurative, diaphoretic, emetic, purgative, diuretic, laxative properties). 
Plant uses other than for treating ciguatera poisoning were also noted when related to the symptomatic effects of ciguatera fish poisoning.

\section{Discussion and Conclusions}

The names of 64 different species reputed to be effective in treating ciguatera poisoning have been presented. These folk remedies are of two types: preventive and curative. For example, the leaves of Ficus spp. (Moraceae) may be used to wrap the fish, or are cooked along with it; alternatively, the fish may be rinsed in an infusion prepared by using a plant, such as Phymatosorus scolopendria (Polypodiaceae). This is claimed to destroy the toxin during cooking.

'Preventive' methods are questionable, since the ciguatera toxin is known to be heat-resistant and water-insoluble.

Among other remedies administered as cures, some are used much more often than others. The following plants are used in remedies that are popular throughout Polynesia and/or Melanesia: Argusia argentea, Artocarpus altilis, Carica papaya, Syzygium malaccense and Vitex rotundifolia.

These remedies consist mostly of single plants and in some cases there is no special preparation involved; the plant is simply eaten in its natural state. When preparation is necessary, three methods are commonly employed: extraction of the juice using a mortar and pestle, infusion and decoction. Dosage is rarely precise. In many cases, a 1-1 bottle of the remedy is prepared, which must be drunk during the course of the day; the quantity is replenished until symptoms disappear.

Remedies are taken internally, except those involving Argusia argentea and Vitex rotundifolia, which may also be used as a bath to relieve itching and Wikstroemia indica which is used externally.

Information on other medicinal uses of the species used to treat ciguatera poisoning is also listed in the table. Eight species are thus also known for their anti-diarrheal, anti dysenteric or antispasmodic intestinal effects. These are: $A c h y$ ranthes aspera, Chenopodium ambrosioides, Euphorbia hirta, Morinda citrifolia, Phymatosorus nigrescens, Sophora tomentosa, Spondias cytherea and Thespesia populnea.

Five species are alleged to have a soothing effect on rheumatism and other pains, including muscle pains and pains of the joints. These are: Argusia argentea, Chenopodium ambrosioides, Entada phaseoloides, Pandanus tectorius, Schinus tereben- thifolius (this one used as a liniment) and Syzygium malaccense.

Other uses of the plants listed include cardiac activity (four species), antiallergic and local analgesic activity (six species), depurative, general detoxifying or diaphoretic activity (nine species).

Considering each species individually, the comparison of its ethnobotanical uses suggests that some species may act on the symptoms caused by the ciguatera toxin and thus have antidiarrheal, antispasmodic, antipruritic or cardiac tonic effects.

On the other hand; pharmacological tests are necessary in order to determine whether or not these plants indeed have direct 'detoxifying' action on the ciguatoxin itself. That is our program's goal, which is to evaluate these remedies' therapeutic potential by studying their effects in mice intoxicated with liver extracts of a carnivorous fish (moray eels) containing ciguatoxin.

\section{Acknowledgements}

We wish to extend special thanks to the men and women of New Caledonia and Vanuatu who welcomed us and offered their time, their patience and their knowledge during our ethnobotanical surveys.

\section{References}

Bagnis, R. (1973) L'ichtyosarcotoxisme dans le Pacifique sud. Commission du Pacifique Sud, Nouméa, NouvelleCalédonie, 112 pp.

Bagnis, R., Chanteau, S., Chungue, E., Hurtel, J. M., Yasumoto, T. and Inoue, A. (1980) Origins of ciguatera fish poisoning: a new dinoflagellate, Gambierdiscus toxicus Adachi and Fukuyo, definitely involved as a causal agent. Toxicon 18, 199-208.

Barrau, J. (1950) Une plante médicinale de la NouvelleCalédonie, Duboisia myoporoides R. Br. Revue Agricole de Nouvelle-Calédonie 1 (7-8), 13-14.

Bidard, J.N., Vijverberg, H.P.M., Freln, C., Chungue, E., Legrand, A.M., Bagnis, R. and Lazdunski, M. (1984) Ciguatoxin is a novel type of $\mathrm{Na}^{+}$channel toxin. Journal of Biological Chemistry 259, 8353-8357.

Bloomfield, S.A. (1986) Is health what we want? A conceptual view of traditional health practices in Tonga with special emphasis on maternal child health and family planning. M.S. Thesis, School of Social Economic Development, University of South pacific, Suva, Fiji.

Bourdeau, P. (1985) Etude des Poissons Ciguatoxiques et de la Ciguatera aux Antilles Françaises. Epidémiologie sur le plateau de St Barthelemy, St Martin, Anguilla. Thèse, Université de Paris Sud, Centre d'Orsay, $289 \mathrm{pp}$.

Bourret, D. (1981) Bonnes plantes de Nouvelle-Calédonie et des Loyautés. Les éditions du lagon, $107 \mathrm{pp}$.

Burkill I.M. (1935) A Dictionary of the Economic Products of the Malay Peninsula. Crown Agents for the Colony, 2 vols. 
Cabalion, P. (1984a) Les plantes du Vanuatu. La 'gratte' et ses remèdes. Première partie: les causes. Vanuatu Weekly Hebdomadaire 12,11 .

Cabalion, P. (1984b) Les plantes du Vanuatu. La 'gratte' et ses remèdes. Deuxième partie: les médications traditionnelles bien connues. Vanuatu Weekly Hebdomadaire 13, 10.

Cabalion, P. (1984c) Les plantes du Vanuatu. La 'gratte' et ses remèdes. Les médications traditionnelles bien connues. Vanuaiu Weekly Hebdomadaire 14, 11.

Cabalion, P. (1984d) Les plantes du Vanuatu. La 'gratte' et ses remèdes. Les médications traditionnelles bien connues. Vanuatu Weekly Hebdomadaire 15, 11.

Cabalion, P. (1984e) Les plantes du Vanuatu. La 'gratte' et ses remèdes. Troisième partie: les médications traditionnelles bien connues. Vanuatu Weekly Hebdomadaire 16, 10-11.

Capra, M. and Cameron, J. (1985) Les effets de la ciguatoxine sur les nerfs des mammifères. Proceedings of the Fifth International Coral Reef Congress, Tahiti 4, 457-461.

Dufva, E., Loison, G. and Holmsted, B. (1976) Duboisia myoporoides, native antidote against ciguatera poisoning. Toxicon 14, 55-64.

Geremie C. (1988) Essai de synthèse des connaissances actuelles sur la Ciguatera. Thèse en Pharmacie, Université de Marseille, $94 \mathrm{pp}$.

Haddock, L. (1973) Quelques Plantes Médicinales de Guam avec Leurs Noms Vernaculaires. Conférence Technique Régionale sur les Plantes Médicinales, Commission du Pacifique Sud, Tahiti, 15 pp.

Lange, W.R., Kreider, S.D., Hattwick, M.M.D. and Hobbs, J. (1988) Potential benefit of tocainide in the treatment of ciguatera. Report on three cases. The American Journal of Medicine 84, 1087-1088.

Legrand, A.M., Lotte, C. and Bagnis, R. (1985) Effets respiratoires et cardio-vasculaires de la ciguatoxine chez le chat. Action antagoniste de l'hexamethonium, l'atropine, le propanolol, la phentolamine, la yohimbine, la prazocine, le verapamil, le calcium et la lidocaine. Proceedings of the Fifth International Coral Reef Congress, Tahiti 4, 463-466.

Legrand, A.M., Galonnier, M., Bourret, D. and Bagnis, R. (1982) Ximenia elliptica, plante de la famille des Olacacées, un antidote contre l'intoxication ciguatérique. Personal communication.

Lobel, P.S. (1979) Folk remedies for tropical fish poisoning in the Pacific. Sea Frontiers 25, 239-245.

Loison, G. (1955) Poisonous fish of the South Pacific, South Pacific Quarterly Bulletin 5 (4), 28-31.
Lombet, A., Bidard, J.N. and Ladzunski, M. (1987) Ciguatoxin and brevetoxins share a common receptor site on the neuronal voltage dependant $\mathrm{Na}^{+}$channel. Federation of European Biochemical Society Letters 219 (2), 355-359.

McCuddin, C. (1974) Samoan medicinal plants and their usage. The Office of Comprehensive Health Planning, Department of Medicinal Services, Government of American Samoa, 35 pp. and annexes.

McMakin, P.D. and Moore, P.H. (1977) A guide to the Medicinal Plants of Guam. Coastal Management Section. Technical Report Bureau of Planning Agana, Guam 2, pp. $1-84$.

Murata, M., Legrand, A.M., Ishibashi, Y. and Yasumoto, T. (1989) Structures of ciguatoxin and its congeners. Journal of American Chemical Society 111, 8929-8931.

Ohizumi, Y. (1987) Pharmacological actions of the marine toxins ciguatoxin and maitotoxin isolated from poisonous fish. The Biological Bulletin 172, 132-136.

Palafox, N.A., Jain, L.G., Pinano, A.Z., Gulick, T.M., Williams, R.K. and Schatz, I.J. (1988) Successfull treatment of ciguatera fish poisoning with intravenous mannitol. Journal of American Medical Association 259, 2740-2742.

Pétard, P. (1986) Plantes Utiles de Polynésie et Raau Tahiti. Editions Haere Po No Tahiti, 353 pp.

Rageau, J. (1973) Les Plantes Médecinales de Ia NouvelleCalédonie. Travaux et documents de l'ORSTOM 23, 139 pp.

Rageau, J. (1983) Fichier des Plantes Médicinales de Tahiti et de Polynésie. ORSTOM Bondy, no pagination.

Vienne, B. (1981-1982) Les Usages Médicinaux de Quelques Plantes Communes de la Flore des Iles Banks (Vanuatu). Cahiers ORSTOM, Série Sciences Humaines XVIII (4), $569-589$.

Weiner, M.A. (1971) Ethnomedicine in Tonga. Economic Botany 25, 423-450.

Weiner, M.A. (1985) Secrets of Fidjian Medicine, 141 pp.

Whistler, W.A. (1985) Traditional and herbal medicine in the Cook Islands. Journal of Ethnopharmacology 42, 155-176.

Whistler, W.A. (1991) Herbal medicine in the Kingdom of Tonga. Journal of Ethnopharmacology 31, 339-372.

Yasumoto, T., Seino, N., Murakami, Y. and Murata, M. (1987) Toxins produced by benthic dinoflagellates. The Biological Bulletin 172, 128-131.

Yokoyama, A., Murata, M., Oshima, T., Iwashita, T. and Yasumoto, T. (1988) Some chemical properties of maitotoxin, a putative calcium channel agonist isolated from a marine dinoflagellate. Journal of Biochemistry 104 (2), 184-187. 